Monatsschrift f. Geburtshülfe u. Gynäkologie 1929;82:147-155

\title{
II. Buchbesprechung
}

Robert Schroder, Der mensuelle Zykíus des Weibes und seine Störungen. (Handbuch der Gynäkologie, L. Band, 2. Halite, 3. völlig neubearbeitefce und erweiterte Auflage des Handbuchs der Gynäkologie von Veil, heraus-gegeben von W. Stoeckel, München. 1928. I. F. Bergmann.

In der Einleitung werden Fortschritte auf dem behandelnden Gebiete seit der letzten Bearbeitung des Handbuches herausgesetzt. Die Menstruation wird als Fehlschlag einer Schwangerschaftsund Fortpflanzungs-arbeit bezeichnet. (Die Herausarbeitung als eine energetische,volumetrísche und dynamische Übung wird aber noch vermißt. Ref.)

Die Wellenbewegung, welche den Frauenorganismus unter gesunden und kranken Verhältnissen durchzittert, ist eigentlich der Kernpunkt des Frauen- und Frauenkrankheitsproblemes. Eine kurze historische Be-merkung über die Entwicklung der Anschauungen bereitet den Boden für die Darstellung des Arbeitsgebietes, wie es sich heute präsentiert, vor. Bei der Begriffsbestimmung des normalen Genitalzyklus wird klar, daß nicht die menstruellen Blutungen, sondern die Prozesse der voraus-gehenden 3-4 Wochen das eigentliche Wesen des Genitalzyklus ausmachen. Praktisch betrachtet muß man den ersten Tag der Regelblutung als den Beginn des neuen Zyklus ansehen. Er ist gewissermaßen der ,,Stichtag”. Ein 28tägiger Zyklus besteht, wenn von diesem ersten Tage der Blutung bis zum ersten Tage der nächsten 28 Tage vei'gehen.

$10^{*}$

148

Buchbespreclrang.

Die erste Regelblutung ist das allgemein anerkannte Signal für die in Ausbildung begriffene Geschlechtsreife. Der Durchsehnitt des Menarche-alters ist 15 1/2 Jahre.

Neben bekannten anderen Faktoren liaben Lebensweise, Beschäftigung, Ernährung, körperliches Wohlbefinden, wie auf Ablauf und Wiederkehr auch auf den Beginn des Zyklus großen Einfluß. Doch ist der erste Men-struationsbeginn von zu vielen in sich wieder komplizierten Faktoren abhängig, als daß zuverlässige einheitliche Zahlen gefunden werden könnten.

Die Zyklusfunktion hört im Durchsehnitt mit 47 Jahren auf.

Sehr interessant sind die Feststellungen des Entwicklungsstadiums des Körpers beim ersten Auftreten des mensuellen Zyklus. Da Länge und Gewicht dauernd bis zum 17. Jahre zunehmen, ist das Körperwachstum im allgemeinen zur Zeit der urn das 15. Jahr eintretenden Menarche noch nicht, wie seither behauptet wurde, abgeschlossen.

Der körperliche Zustand bei der ersten Menstruation zeigt wohl eine gewisse Entwicklung an, entspricht aber noch nicht dem einer vollerblühten Jungirau.

Am Becken speziell setzt um die Jahre 11-15 ein erhebliches Wachstum ein, aber ein offenbarer Raiimzuwachs findet noch bis zum 16. und 18. Jahre statt. Der soziale Einfluß auf das Beckenwachstum ist deutlich. Diejenigen Mädchen, die körperliche Arbeit leisten und gleichzeitig gute Ernährung haben, sind in der Beckenausbildung voraus. Für die Mehrzahl der Mädchen wird erst um das 17. Jahr vom Becken das geburtshilflich praktisch not-wendige 
Raummaß im wesentlichen erreicht. Auch in beziig auf die Brüste-entwicklung ist zu erkennen, daß die erste Regelblutung erst dann erseheint, wenn ein gewisser Abschluß in der Entwicklung des weiblichen Geschlechts-charakters eingetreten ist. Sehr schöne Abbildungen über die Entwicklung des Uterus im Kindesalter finden sich auf einer Vergleichstafel Seite 33, wie überhaupt der Bilderschmuck des Werkes ein sehr reicher und aus-gezeichneter ist.

An den Primärfollikeln des kindlichen Eierstockes geht ein Wachstum bis zu einem gewissen Grade vor sich. Döch bricht die Entwicklung in einem Punkte ab, worauf die Follikelatresie folgt. Sie ist charakteiïsiert durch den Untergang des Eies und der Follikelepithelien sowie den Ver-schluß des entstandenen Gewebsdefektes. Es liegt kein Grand vor, diese Formationen als eine Eierstocksdrüse zu bezeichnen, wenn auch feststeht, daß lediglich die Keimzellen und deren Abkömmlinge, die Follikelepithelien, als die Träger der Inkretion angesehen werden können. Der Uterus wächst, wie die prächtige Serie von Schnitten durch das Genitale praktisch zeigt, erst vom 10. Jahre ab über seinen Initialziistand hinaus.

Das Eizellenmaterial, das während des Kindesalters besonders in der letzten Zeit verbraucht wird, ist der Träger der Hormone, durch welche die Entwicklung zur Geschlechtsreife verursacht wird. Der Weg hirer Wirkung geht mit Wahrscheinlichkeit über Hypophyse und Schilddruse. Den größten Anteil an dem zyklischen Prozeß des weiblichen Genitals nehmen Ovarium und Uteruskörper. An der Cervix uteri fehlen sicher nach-weisbare Zyklen. An der Tube läßt sich einiges zeigen. An der Scheide be-findet sich möglicherweise ein Zyklus. Schilddrüse,

Hypophyse, Nebenniere zeigen dem Zyklus des Genitales synchrone Veränderungen.

Buehbesprechung. 149

Durch die zyklischen Veränderungen des Ovariums wird die Fort-pflanzungsarbeit möglich, wenn das Ei und mit ihm sein Follikel zum ersten Male über sein bisheriges

Entwicklungsstadium hinauswächst und Veränderungen durchmacht, die eine letzte und wichtigste Vorbereitung für die Befruchtung und damit die Fortpflanzung bedeutet: die Eireifung. Die wesentlichsten Prozesse offenbaren sich durch eine erhebliche Ver-größerung des Eies und eine Lockerung und bestimmte Formierung des Chromosonenapparat.es sowie eine Reduktíon des Chromatins. Das Ei mitsamt den es unmittelbar umgebenden Follikelzellen tritt aus und wird mit dem ausfließenden und herausgepreßten Liquor in die Bauchhöhle oder direkt in die Tube , geboren".

Der bei weitem größere Anteil der Zellen aber bleibt in gutem Zustande und macht im Verein mit der Theca interna sehr bald eine bemerkenswerte Umwandlung durch, die in wenigen Tagen zum Aufbau einer typischen endokrinen Druse, der Granulosadrüse (Aschoff) oder dem Corpus luteum, führt,

Das Bedeutsamste an der Granulosadrüse ist ihre zeitliche Begrenzt-heit. Sie blüht rasch in 3-4 Tagen zur vollen Größe und Funktion auf, bleibt etwa 8-10 Tage so erhalten und verfällt, wenn keine Schwanger-schaft eintritt, einer rasch fortschreitenden Schrumpfung und Vernarbung. Die letzten 3 Tage vor der Menstruation ist die Granulosadrüse auf dem Höhepunkt ihrer Entwicklung angelangt. Ist jetzt eine Befruchtung des aus einer Granulosadrüse entstammenden Eies eingetreten, so geht das Wachstum der Granulosadrüse in dem Sinne weiter, daß ihre Zellen noch größer werden und das ganze Gebilde noch hyperhophischer und saf tiger wird.

Ist aber das zugehörige Ei nicht befruchtet, sondern geht es zugrunde, dann setzt jetzt in der Granulosadrüse ein rasch fortschreitender Ver-fettungsprozeß ein.

Mit der Beschreibung der zyklischen Veränderung am Uterus wird ein Spezialgebiet von Schroder betreten. Es ist zweifellos Robert Schröders Verdienst, die Bedeutung der zyklischen Veränderung erkannt und ins-besondere ihre charakteristischen Bilder herausgearbeitet zu haben. 
Es ist das Verhalten der Uterusschleimhaut zu Zeiten der Periode recht klar herausgesetzt worden, und er hat uns die Bilder der funktionellen hypo-plastischen Prozesse von denjenigen der echten Enolomehitis unter-scheiden gelehrt.

Nach Schröders Untersuchungen ist der Zykhis im Endometrium eine regelmäßige physiologische Erscheinung, die sich auch bei pathologischen Affektionen, wie Myom, Karzinom, Ovarialtumor, chronischen entzünd-lichen Prozessen, Lageanomalien usw., ebenso feststellen läßt, wie unter normalen Verhältnissen.

Wichtig ist, daß bis jetzt der Nachweis von. Nerven im Endometrium noch nicht gelungen ist. Sollte sich das bestätigen, so ware damit der Nachweis erbracht, daß auch ohne unmittelbare Nerveneinwirkung weit-gehende Zellumwandlungen auf hormonalem Wege möglich sind. Die zyklischen Veränderungen des Endometriums bestehen in den wesentlichsten Punkten darin, daß die oberflächliche Partie einer niedrigen ruhenden Schleimhaut eine sehr erhebliche Auflockerung und ein charakte-ristisches Wachstum erfährt, daß in dieser durch Proliferation eines Teiles des Endometriums entstandenen neuen Schicht Sekretionsvorgänge auf150

Buchbesprechung.

treten, und daß auch das Bindegewebe sich an dieser Polster- oder N-est-bildung beteiligt. Diese neugebildete und besonders umgewandelte Schicht ist die Einnistungsstätte eines neuen Embryo. Sie nimmt ihn auf und bildet an den von der Implantationsstelle nicht eingenommenen Partien die in der Schwangerschaft sogenannte Decidua. Kommt ein Embryo nicht zur Ausbildung, findet also keine Implantation auch nicht außerhalb des Uterus statt, so tritt eine Sequestration und völlige Destruktion der besonders gebildeten Schleimhautschicht ein. Der Rest des Endometriums erhält sich. Nur ein Teil des vorhandenen Endometriums, und zwar der obere, beteiligt sich an der Bildung der neuen Schicht, und nur diesem Teil fallen die Zyklusveränderungen zu, während der tiefer gelegene Abschnitt im wesentlichen keine Veränderung erfährt. Es geht daraus die Notwendig-keit hervor, diese beiden Teile besonders zu benennen. Die tiefer gelegene lieißt dieBasalschicht, die den Zyklus bildende, höher gelegene dieFunktions-oder Nidationsschicht. Alle diese Vorgänge sind durch gute Bilder illustriert. Über den Isthmus uteri läßt sich sagen, daß eine wenn aucb nur rudimentäre Reaktion und damit eine gewisse Empfangsbereitschaft für das Ei in seinem Bereiche auftritt, also eine tatsächliche Beteiligung dieser Stelle am Zyklus stattfindet. An der Tube besteht eine zweifellose, wenn auch schwer nachweisbare Beteiligung am Zyklus.

Für die Gründlichkeit des Schröderschen Buches spricht der Versuch, auf alle mòglichen Gebiete seine Ideen vom mensuellen Zyklus auszubreiten und dort bestätigt zu finden. Zur Erörterung der Phylogenese des Genital-zyklus werden Säugetiere und Wirbeltiere, von den Fischen bis zu den Vögeln, herangezogen. Aus dieser außerordentlich mühsamen Unter-suchung ergab sich, daß überall eine Periodizität der Genitalfunktion besteht. Der Höhepunkt der Genitalfunktion wird durch die Brunst dar-gestellt. Eine Zeitspanne der Manifestation des Paarungstriebes, verbunden mit Steigerung des gesamten somatischen Geschehens im Tierkörper, die Brunstzeit, ist gleichzeitig die einzige Phase, in der das Weibchen die Begattung duldet. Zu jeder anderen Zeit wehrt das Weibchen das Männchen ab. Bei den Primaten ist diese scharfe zeitige Umgrenzung des Paarungstriebes verlorengegangen, sie sind jederzeit zur Kohabitation bereit. Am Genitale laufen anatomische Veränderungen synchrom mit den äußerlich sichtbaren Manifestationen der Sexualtätigkeit ab, die einen Kreisprozeß darstellen, da sie nach einer gewissen Zeit stets ins Ausgangsstadium zurückkehren. Es empfiehlt sich, für die Vergleichsbetrachtung die ein-zelnen Phasen nach besonderen Gesichtspunkten festzulegen: 
1. Proliferation; 2. Destruktion; 3. Regeneration. Alle diese Vorgänge

gruppieren sich um den im Mittelpunkt stehenden Prozeß der Ovulation.

Bei diesen Untersuchungen stellt es sich heraus, daß die morphologischen Stadien (Proliferation, Destruktion, Regeneration) mit den seither gebräuch-zeitlichen Phasen (Proöstrum, Ostrum, Met rostrum) sich weitgehend decken.

Die Untersuchung der zeitlichen und ursächlichen Zusammenhänge im Ablaufe des Zyklus ergab: 1. Die Proliferationsphase des Endometriums entwickelt sich nur, wenn gleichzeitig im Ovar ein reifender Follikel vor-h and en ist.

2. Eine Sekretionsphase ist bisher nur dann naehgewiesen worden, wenn gleichzeitig ein Corpus luteum besteht. Je weiter die Entwicklung Buchbesprechuug. 151 des Corpus luteum vor sich geht, um so mehr nimmt auch das Sekretions-stadium an Ausbildung $\mathrm{zu}$.

Zur Zeit der Desquamation und Sequestration der Uterusmukosa zeigt das Corpus luteum eine starke Zunahme der nachweisbaren Lipoide, und die Rückbildung macht von jetzt ab rasche Fortschritte.

Nach erfolgter Epithelialisierung und beginnender Neuproliferation findet sich dann wieder der neue reifende Follikel, während das Corpus luteum weiterer Rückbildung anheimfällt.

Alle diese Vorgänge sind durch vorzügliche Schemata erläutert.

Die Brunst der Tiere entspricht der Ovulation des Menschen und nicht der Menstruation. Eine Menstruation in dem Sinne, wie sie beim Menschen notwendigerweise aufgefaßt werden muß, mit den beschriebenen anato-mischen Substraten im Ovar und Uterus, insbesondere mit der die Blu-tungen bedingenden Desquamation der Murosa, findet sich nur bei Affen und Menschen, das heißt bei den Tieren, bei denen eine innigere Plazentation, wie Grosser sagt, hämochoriale Plazentation stattfindet und der Fötus sich einen intervikösen Raum schafft. Bei den übrigen Tieren verläuft die Granulosadegeneration und das Abschwellen der Schleimhaut ohne klinisches Zeichen. Sie ist also für den klinischen Beobachter nicht feststellbar.

Definieren wir jedoch die Menstruation in dem bisher immer wieder angegebenen Sinne, daß nach vorausgegangenem Ovarial- und Uterus-zyklus eine Sequestration bei ausgebildeter Uterusschleimhaut und eine Rückbildung des Corpus luteum eingetreten ist, so muß unbedingt fest-gestellt werden, daß eine Menstruation während der Schwangerschaft nicht eintreten kann, da die Uterusschleimhaut ja von einem in Ent-wicklung und Wachstum begriffenen Ei völlig in Anspruch genommen ist.

In der Mehrzahl der Fälle, also in der Norm, ist der Ubergang des reifen Follikels in die frische Granulosadrüse bei regelmäßig vierwöchent-lichem Zyklus um den 14.-16. Tag anzusetzen. Er ist aber ausnahmsweise auch zu jeder anderen Zeit durch die Verschiebung der Eireife im Sinne einer Verzögerung oder Beschleunigung der Ovulation möglich.

Die günstigste Konzeptionsgelegenheit liegt in der zweiten Zyklus-woche. Immerhin kann Konzeption zu alien Zeiten des Zyklus befruchtend wirken. Zur Erklärung bestehen zwei Möglichkeiten, entweder die Ovulation ist zu jeder Zeit des Zyklus möglich und ist im wesentlichen abhängig von dem hyperämisierenden und nervösen Einflüssen der Kohabitation. Oder es bleibt nichts anderes übrig als die Annahme zu machen, daß entweder die Spermatozoen auf das Ei oder umgekehrt das Ei auf die Spermatozoen wartet.

Die Annahme vom raschen Zugrundegehen der Spermatozoen ist durch eine Reihe von Erfahrungen widerlegt, und es scheint der Auffassung, daß die Spermatozoen mehrere Tage warten können, tatsächlich nichts im Wege zu stehen. 
Vorerst spricht alles für einen allgemein festliegenden spontanen Ovulationstermin, und zwar in der Mitte des Zyklus.

Die Ursache der Follikelreifung ist das stets nachzuweisende reifende Ei. Das Blütestadium der Granulosadrüse geht in langsameni Fortschreiten seiner inneren Organisation völlig parallel mit einem ebenfalls langsamen Zunehmen der Drüsensekretion und einer Umwandlung zu einem unmittel-

152 Buchbesprechung.

bar prägraviden Stadium. Der Übergang in die Schwangerschaftsverände-rung ist absolut fließend.

Wenn aber das Ei tatsächlich dominiert, welche Rolle hat dann die Granulosadrüse überhaupt ? In der phylogenetischen Untersuchung war zu sehen, daß eine solche Granulosadrüse sich erst bei den Tieren findet, die lebendige Junge zur Welt bringen und die eine innigere Beziehung des Keimlings mit der Mutter zeigen, insbesondere bei den Plazentaliern.

Die Granulosadrüse scheint deshalb im Zyklus notwendig zu sein, weil die zu treffenden Vorbereitungen durch das winzige Ei allein nicht erregt werden können. Das Ei braucht eine leistungsfähige Hilfe. Ebenso wie das reifende Ei auch nicht allein für die Proliferation der XJterus-schleimhaut sorgen konnte, sondern dazu die Mitwirkung der Follikel-epithelien in der Membrana granulosa seiner Eiblase benutzt, ebenso reagiert auch die Granulosaluteinzelle mit offenbar größter Empfindlichkeit auf Stoffe, die von der Eizelle ausgehen und ihr Leben anzeigen. Es handelt sich also urn eine Verstärkungsdrüse. Schroder hat das als Relaiswirkung bezeichnet. Offenbar handelt es sich um eine Art Multiplikator oder Kraft -übersetzung (Ref.). Als Triebstoffe für diese Prozesse kommt das Ovarialhormon in Be-tracht; Stoffe, welche Nidationsbettbildtmg, also den Uterinenzyklus, oder, auf das Tier übertragen, den Brunstzyklus herbeiführen können, sind sowohl aus dem gesamten Ovarium, wie aus dem Corpus luteum, als auch aus der Plazenta zu extrahieren. Daß auch die Plazenta solche Stoffe ab-sondert, braucht einen nicht zu wundern. Die Plazenta enthält Fötmaterial, das den jüngsten Entwicklungsstadien des Eies entstamrnt, und die IГol-likelepithelien sind ebenfalls Eiabkömmlinge. Plazenta und Föt treten lediglich für das Corpus luteum als Stimulans für die Genitalwege ein. Die XJntersuchungen von Zondek und Heyn haben gezeigt, daß die von ihnen dargestellten Ovarialhormone sowohl als Wachstumsstimulans, wie auch als Brunsthormon wirken. In jüngster Zeit sind auch andersartige unspezifische, brunsterzeugende Stoffe festgestellt worden, z. B. Extrakte aus Fruchtknoten und Blüten-substanz.

Bei der strikten Reihenfolge der Aufeinanderfolge der Eireifungen darf auf eine gewisse Behinderung eine Hemmung der Follikelreifung durch die reife Granulosadrüse geschlossen werden. Ein reifes Ei duldet offenbar keinen andern reifen Follikel neben sich. Etwas, was ich als Dominanz-wechsel bezeichnet habe (Ref.). Es kann entgegen früheren Anschauungen nur dann von einer Menstruation gesprochen werden, wenn die Granulosadrüse in beginnender Rückbildung und eine vorher prägravid gewesene Schleimhaut in Sequestration sich befindet. Die klinischen Folgezeichen bei der durch die Sequestration entstandenen Wundfläche sind Blutungen aus den zerrissenen Gefäßen. Es ist wie bei einem Abortus oder Partus, nur in stark verkleinertem Maßstabe.

Man hat gemeint, daß primär gewisse dem Ei übergeordnete Faktoren bestehen. Man hat diese Herrschaft in der Hypophyse gesucht.

Sehen wir das zyklische Geschehen in dem Sinne einer Schwanger-schaftsvorbereitung mit der Bereitschaftsstellung zur Befruchtung an, so ist es plaxisibel, daß nicht nur im Genitale, sondern auch darüber hinaus in den verschiedensten Organsystemen des Körpers Funktionsänderungen 
Buchbesprechung.

153

vor sich gehen, die denen ähnlich sind, wie sie in vergrößertem Maßstabe während der

Schwangerschaft durch Umstellung auf die ganz besonders hochwertige Fortpflanzungsfunktion des Körpers zustande kommen. Es ist das gemeint, was man früher eine Wellenbewegung der körperlichen Funktion der Frau genannt hat.

Beim Ausstoßungsmechanismus des Menstrualblutes ist zweifellos, daß er durch Muskelkraft bewirkt wird. Sehr interessanterweise hat Stieve durch Messung der Muskelzellen des Myometriums feststellen können, daß diese während und nach der Blutung kleiner werden und an Masse verlieren, wie während der Geburt und im Wochenbett; und daß sie im Verlauf des Zyklus dann wieder eine Massenzunahme bis zum Beginn der nächsten Regel durchmachen. Auch das Bindegewebe begünstigt die Vor-gänge durch Auflockerung und Wiederbefestigung. Also immer mehr Ähnlichkeit zwischen dem kleinen und großen Funktionsgang des weib-lichen Sexualapparates (Ref.)!

Durch die Menstruation schlägt eine normale Scheidenflora für wenige Tage in eine bunte Mischflore um.

Eingehende Besprechung erfahren die klinischen Begleiterscheinungen des Zyklus im übrigen Körper, ohne daß darauf aber in diesem Referat weiter eingegangen werden könnte. Sehr bemerkenswert ist auch das Ver-halten des Seelenlebens während der Menstruation, doch scheint hier die psychische Veranlagung das Wichtigste zu sein.

Die Dietätik in bezug auf den mensuellen Zyklus ist sehr eindringlich geschildert. Die Frau trägt zur Zeit der Menstruation eine Wundfläche in sich. Es ist zweifellos, daß in dieser Zeit eine Infektionsmöglichkeit ge-geben ist. Es sind auch tatsächlich intramensuelle Peritonitiden beobachtet worden.

Das Klimakterium wird als die natürliche Menopause bezeichnet. Besonders wertvoll sind die Ausführungen uber das Verhalten des übrigen Körpers beim natürlichen Ausschalten der Genitalfunktion. Uber die Hälfte der Frauen kommen ohne lästige Beschwerden durch die Wechseljahre hindurch.

Der zweite Teil des hochinteressanten Buches befaßt sich mit den Störungen des mensuellen Zyklus.

Macht man sich los von alien Vorurteilen und Voreingenommenheiten, so ist die Schwangerschaft der natürliche Endzweck des im ersten Teile besprochenen Funktionsganges, dagegen der Abschluß mit dem Nieder-bruch ohne Fruchtobjekt, eben die menstruellen Blutungen, die Ausnahme.

Die fehlende Befruchtungsgelegenheit, die absichtliche Befruchtungs-verhütung und viele andere Ablenkungsmomente, wie sie die Domesti-kation und die Erfordernisse und Einwirkungen des kulturellen Lebens herbeiführen und verlangen, haben eine Umwertung in den Vorstellungen geschaffen, so daß wir heute den Fehlschlag eines Funktionsganges, die menstruelle Blutung, als das gewöhnliche, das normale, die Erfüllung dieser Funktion, die Schwangerschaft, jedoch als die Ausnahme ansehen. Trotzdem nimmt für die Darstellung Schroder den Standpunkt ein, wie sich die Prozesse für den Kulturmenschen gestaltet haben. Die Konstitution ist von großem Einfluß auf den Ablauf des Zyklus.

Eine bedeutende Wirkung auf krankhafte Störungen der Regel hat 154

Buchbesprechung. 
nach aller Erfahrung die erste Schwangerschaft mit Geburt und Wochen-bett, indem vorherige Unregelmäßigkeiten regelmäßig, aber auch Regel-mäßigkeiten unregelmäßig werden können, auch die Blutungstärke eineVer-änderung erfährt. In der überwiegenden Mehrzahl der Fälle ist jedoch eine wesentliche Änderung für diese normalen Vorgänge nicht zu konstatieren. Je starker die äußere Einwirkung auf den Körper ist, um so bedeut-samer ist häufig auch die folgende Zyklusveränderung. So wird nach schweren Unfällen von Regelstörungen berichtet. Die tägliche Krankenhaus-erfahrung lehrt, daß auch durch Operationen der Zyklus, sowohl in Tempo wie in Stärke, verändert werden kann. Akute Infektionskrankheiten, chronische Infektionskrankheiten, Zirkulationsorgane und Gefäßsystem zeigen mancherlei Einfluß auf den Ablauf des Zyklus, ebenso Erkrankung der Verdauungsorgane, Lebererkrankungen, Nierenerkrankungen, Stoff-wechselerkrankungen, Bluterkrankungen und Störung der endokrinen Drüsen. Viel ist über die psychische Einwirkung auf Ablauf des Zyklus geschrieben worden. Den Schluß bildet ein ausgezeichnetes Resume über die Beziehungen der Erkrankungen des Körpers zum Ablauf des mensuellen Zyklus.

Nun folgt die Feststellung, wie sich der mensuelle Zyklus bei genitalen Erkrankungen verhält: bei Hypoplasien und Lageanomalien, entzünd-lichen Erkrankungen, Mißbildungen, Ovarialtumoren, Myomen. Nach dieser Übersicht über die Einwirkung krankhafter Vorgänge auf deń Ablauf des mensuellen Zyklus wird das große Gebiet der Zyklusstörungen im einzelnen durchgesprochen: das Auftreten des Zyklus in außerphysiolo-gischen Zeiten, die Menstruation des Neugeborenen, die Menstruatio praecox, die verspätete Menarche, die verfrühte Klimax, die spate Menopause, zyklische Blutungen während der Schwangerschaft, Laktation und Zyklus. Ein weiteres Kapitel behandelt das Ausbleiben des Zyklus in der Geschlechtsreifezeit, Pseudoamenorrhöe, Kastration, die echte iVmenor-rhöe. Unbestritten ist, daß die Konstitution des Körpers für den Ausfall der Ovarialfunktion und dessen Prognose eine wichtige Bedeutung hat. Zur Heilung verwendet man neben Eierstockssubstanzen, wie sie irgendwie aus dem Organ hergestellt werden, auch die Überpflanzung von Eierstocks-gewebe. Durch die Auswertung der Funktionsänderung zu den andern Organen bei Ausfall der Eierstocksfunktion ist es berechtigt, auf den Umweg über diese Organe die Funktionsfähigkeit der Ovarien wieder zu bessern. Hier kommen Schilddrüse, Hypophyse und Bauchspeicheldrüse in Betracht.

Ein anderer großer Abschnitt betrifft die Abweichungen im Zyklus-tempo. Es ist hier von einer primären und sekundären Schwäche des Keim-plasmas die Rede. Dann kommen die morphologisch faßbaren Störungen des Keimplasmas und ihre Auswirkungen auf den Ablauf des mensuellen Zyklus. Besondere Bedeutung erlangt die pathologische Tendenz reifender Follikel. Wie aus der anatomischen Beschreibung hervorgeht, handelt es sich um ein gut charakterisiertes Krankheitsbild, das im Ovarium Follikel zeigt und im Endometrium als Folge dieser abnorm ausgedehnten Follikel-wirkung ein ins Pathologische gesteigerte glanduläre Proliferation mit lokal begrenzten, oft allerdings ziemlich ausgedehnten Zirkulationsstörungs-bezirken aufweist. Robert Schroder schlägt vor, diese Krankheit als Metro-

Buchbesprechung. ]55

pathia haemorrhagica zu bezeichnen. Die ganze Beweisführung ist durch vorzügliches illustratives Material gestützt.

Besonderes Interesse erweckt auch noch der Abschnitt Pathologisches vom Corpus luteum (Granulosadrüse).

Der dritte Hauptabschnitt befaßt sich mit den funktionellen Störungen des Uterinenzyklus. Es werden die zu schwache Regelblutung, die zu starke Regelblutung abgehandelt. Dann kommen die morphologisch faßbaren Störungen des uterinen mensuellen Zýklus. 
Hier soil nur aufmerksam gemacht werden auf die Entzündungen im Corpus uteri und ihre Bedeutung für den Zyklusablauf, die eigentliche Endometritis corporis. Ein hübsches Schema erläutert die Zusammenhänge.

Auch die überall eingestreuten Blutungskurven wirken sehr instruktiv. Tuberkulöse Endometritis, Endometriosen (heterope Endometriuminseln) und Blutungen aus dem senilen Uterus sind einige besonders aufgeführte Krankheitsbilder.

Die abnormen Begleiterscheinungen des Zyklus bilden den Abschluß des Buches. Abnorme lokale Erscheinungen im Anschluß an die Regel, alle möglichen Beschwerden zwischen den Regeln , unregelmäßige Blutungen, viele besondere Krankheitsbilder finden ihre Erörterung. Vikarierende Blutungen gibt es nicht, ebensowenig wie komplementäre. Die beschriebenen Fälle sind lediglich eine Gruppe unter den sonstigen Organblutungen auch. In den Ausführungen über die schmerzhafte Regel findet sich alles zusammengestellt, was man über dieses praktisch so bedeutsame Kapitel sagen kann.

Ein Ill Seiten langes Literaturverzeichnis bildet den Schluß dieses außerordentlich wertvollen und neuartigen Buches.

Robert Schroder hat als oberstes Gesetz im Frauenkörper den men suellen Zyklus und seine Störung herausgearbeitet und diese fortwährende Frauenschwankung, die das Wesen der Frau ausmacht, endlich einmal biologisch und anatomisch richtig gefaßt. Es liegt ihm ganz von selbst nahe, möglichst vieles vom normalen Verhalten der Frau und ihren Krankheiten auf dieses oberste Gesetz zurückzuführen oder wenigstens mit ihm in Einklang zu bringen. Damit ist freilich eine Art Neuordnung der gesarnten Frauenheilkunde angebahnt, die ja Robert Schroder selbst in seinem Lehrbuch der Frauenkrankheiten sowieso schon durchzuführen gesucht hat. Robert Schroder geht nicht weiter, als ihm seine anatomischen und literarischen Beweisstücke zuverlässige Stütze sein können. Wir müssen dem Autor für seine ungeheuer mühevollen, aber auch von größtem Erfolg gekrönten Ausführungen außerordentlich dankbar sein. Das Buch zu lesen und zu erfassen, ist wirklich eine Lust. Es hat etwas geradezu Befreiendes auf dem doch vielfach stagnierenden Gebiete unserer Frauenwissenschaft, einen solchen neuen Lebenshauch zu verspüren. Hoffentlich erleben recht viele von den Fachgenossen diesen Genuß, den einem ein gründliches Studium dieses prächtigen SchrOderschen Werkes verschafft. Bei diesem Kapitel hat der Herausgeber des Handbuches einmal wieder den richtigen Mann zu dem ihm liegenden Stoff gefunden.H. Sellheim. 